\title{
PLATAFORMAS DE MÚSICA ONLINE Práticas de comunicação e consumo através dos perfis ${ }^{1}$
}

\author{
Adriana Amaral ${ }^{2}$
}

\begin{abstract}
Resumo: $O$ artigo tem como objetivo analisar as práticas de consumo $e$ conteúdo musical gerado nas plataformas sociais de distribuição, classificação, recomendação e divulgação de música na Web. Num primeiro momento, levantamos um histórico conceitual no qual discutimos as definições e características desses sites de redes sociais. Posteriormente descrevemos essas características e fluxos comunicacionais nas práticas dos usuários em uma observação inicial comparativa entre três plataformas (Last.fm, MySpace, Blip.fm). Consideramos a emergência de algumas categorias de análise: o papel das recomendações e das classificações dos gêneros musicais no Last.fm; o caráter de consciência sobre a audiência segmentada no MySpace; e a constituição de uma reputação por microconteúdos musicais no Blip.fm.
\end{abstract}

Palavras-Chave: Plataformas de música online. Consumo. Sites de Redes Sociais.

\begin{abstract}
This paper main goal is to analyze the consumption practices and musical content generated on web-based social plataforms of musical distribution, classification, recommendation and release. On the first part we make a distinction between the current literature definitions and main caracteristics of this specific social networks sites. On the second part, we watch and describe this caracteristics and communicational flows in the practices of their users, comparing three different plataforms (Last.fm, MySpace, Blip.fm). Our analysis is based on three emergent categories: the role of recommendation and classification of musical genres at Last.fm; the consciousness niche audience of MySpace users; and the constitution of a reputation through musical microposts at Blip.fm.
\end{abstract}

Key-words: Online Music Plataforms. Consumption. Social Network Sites.

\section{Consumo musical e estilos de vida através dos perfis online}

Os estudos sobre as relações de circulação e consumo midiático no contexto dos estilos de vida na sociedade contemporânea tem apontado os efeitos desse amplo

\footnotetext{
${ }^{1}$ Esse artigo é uma versão ampliada e revisada do trabalho apresentado no Grupo de Trabalho "Comunicação e Cibercultura”, do XVIII Encontro da Compós, na PUC-MG, Belo Horizonte, MG, em junho de 2009.

${ }^{2}$ Doutora em Comunicação Social pela Pontifícia Universidade Católica do Rio Grande do Sul, PUCRS, com Estágio de Doutorado pelo Boston College, EUA. Docente e pesquisadora do Mestrado em Comunicação e Linguagens da Universidade Tuiuti do Paraná (UTP) e do curso de Comunicação Social da Faculdade Internacional de Curitiba (FACINTER). E-mail: adriamaral@yahoo.com
} 
universo de bens simbólicos e cultura material disponíveis atualmente. O processo de estetização da vida cotidiana, em curso desde pelo menos o século XVII e tornado mais visível pelos meios de comunicação de massa no século XX, continua a configurar padrões identitários através de perfis de consumo, seja num contexto amplo dos lazeres e da produção massiva de objetos, vestuários, bens simbólicos; seja em um âmbito mais específico dos grupos e subgrupos a eles interligados.

Os padrões de consumo definiram ou ajudaram a definir a identidade de diversas subculturas, como a dândi, a boêmia e até mesmo a "apache". De maneira semelhante, eles definiram as "tribos" britânicas do fim do século XX: os mods, os rockers, os skinheads, os punks e assim por diante. Também passou a existir uma política de consumo ou, mais especificamente, uma recusa em consumir certos produtos.” (BURKE, 2008: 34)

No âmbito da cibercultura, uma faceta notadamente marcada desses fenômenos acontece através das práticas de construção de perfis online em redes de relacionamento. Esses processos de subjetivação e consumo nos perfis dos sites de redes sociais são constituídos em um contexto macro.

As pessoas se apresentam à rede pelos perfis e pelas páginas personalizadas nas quais compartilham suas preferências. É pela fruição de bens culturais que é constituída a mediação entre os usuários, e ela é resultado de práticas de consumo. Essa fruição, porém, não é apenas índice de identidade ou estilização de vida, status ou capital cultural. Os bens estão ali também para circulação, não importando mais de quem e de onde partiram e ao que se destinam. As atividades de circulação de perfis pela rede tornam cada vez mais complexo este usuário-consumidorprodutor atravessado por uma contagiante e viral pluralidade de preferências e estilos.(PINHEIRO, 2008: 106)

Em estudos anteriores indicamos algumas práticas comunicacionais e sociais através da constituição de perfis específicos de uma cena e estética musical no MySpace (2007b), bem como apontamos as práticas subculturais como elementos históricoconceituais na gênese da cultura digital e que retornam a um lugar de importância 
através da popularização das redes de relacionamento (2008). Já Liu (2007) observa esses perfis/estilos de vida enquanto performances de gosto, nas quais ele encontra quatro categorias: prestígio (reputação), diferenciação, autenticidade e persona teatral.

One of the newest stages for online textual performance of self is the Social Network Profile (SNP). The virtual materials of this performance are cultural signs-a user's self-described favorite books, music, movies, television interests, and so forthcomposed together into a taste statement that is "performed" through the profile. By utilizing the medium of social network sites for taste performance, users can display their status and distinction to an audience comprised of friends, co-workers, potential love interests, and the Web public. (LIU, 2007: Online)

No caso específico da formação de um "gosto musical”3, complementar às possibilidades midiáticas massivas como o rádio, a televisão, os jornais, as revistas etc., os perfis online em redes de relacionamento tem se mostrado eficientes e vigilantes no sentido de constituição de um banco de dados de consumo, de memória musical, de organização social em torno da música, de crítica musical e classificação de gêneros, de constituição de reputação de conhecimento sobre o assunto e, quando aliados aos sistemas de recomendações musicais como no caso do Last.fm ${ }^{3}$, essas possibilidades “ultrapassam os limites da área de recuperação de informação (...) pois a recomendação per se é, antes de tudo, fruto de um processo social e tem influência dos elos sociais estabelecidos ao longo da atuação humana nesse processo”. (Figueira Filho, Geus e Albuquerque, 2008: Online)

O presente artigo apresenta um comparativo entre três plataformas sociais de música online: Last.fm, MySpace ${ }^{4}$ e Blip.fm ${ }^{5}$, a fim de observar as estratégias de consumo e classificação de conteúdo gerado pelos fãs-usuários a partir desses sites de relacionamento. Partimos de um breve histórico e da observação-participante nas plataformas e de dados empíricos (no caso da Last.fm) ${ }^{6}$, no qual analisamos algumas

\footnotetext{
${ }^{3}$ http://www.last.fm. Acesso em 06/01/2009

4 http://www.myspace.com Acesso em 10/01/2009.

5 http://www.blip.fm Acesso em 10/01/2009.

${ }^{6} \mathrm{O}$ questionário sobre as práticas de social tagging dos usuários brasileiros do Last.fm foi elaborado conjuntamente com Maria Clara Aquino (UFRGS) e disponibilizado online do dia 19/01/2009 ao dia 05/02/2009 no endereço http://spreadsheets.google.com/viewform?key=pH1GnL4IJw6vIsgS5aMtpcg e distribuído via Twitter, blogs, listas de discussão de música e pelos próprios perfis de amigos e vizinhos
} 
práticas comunicacionais cotidianas dos usuários nessa rede, destacando o papel das recomendações e das classificações dos gêneros musicais no Last.fm; o caráter de mediação social e laços de “amizade” entre os fãs no MySpace; e a constituição de uma reputação por micropostagens musicais no Blip.fm.

Não pretendemos aqui esmiuçar as questões relativas às relações de visibilidade e vigilância - Sibilia (2007) e Bruno (2008), respectivamente, apresentam análises sob essas óticas - intrínsecas a processos nos quais o público e o privado se mesclam e alternam. Nosso enfoque apresenta relações entre a própria materialidade dos sites observados em relação aos usos e apropriações de seus usuários enquanto ferramenta de entretenimento e de disseminação de informações musicais. Consideramos a produção e a classificação de conteúdo musical gerado pelos usuários (artistas/fãs) em rede enquanto elemento de arquivamento informativo da memória musical, consciência de audiência segmentada em termos de identidade online.

\section{Plataformas de música online - breve histórico, estudos e definições}

Social networks, a very old and pervasive mechanism for mediating distal interactions among people, have become prevalent in the age of the Web. With interfaces that allow people to follow the lives of friends, acquaintances and families, the number of people on social networks has grown exponentially since the turn of this century. Facebook, LinkedIn and MySpace, to give a few examples, contain millions of members who use these networks for keeping track of each other. (Huberman, Romero e Wu, 2008: Online)

Devido ao intenso crescimento e popularização dos sites de redes sociais, uma das tendências de apropriação foi a segmentação em nichos de "gosto" e estilos de vida, como redes de relacionamentos voltadas a animais domésticos (como o Orkupet), moda (MySpace Fashion), atuações profissionais (por ex. Linkedin), de música, entre outros. "Many newer social network sites are highly specialized, targeting specific user groups such as Christians, the elderly, knitters, or movie fans” (Baym \& Ledbetter, 2008: Online).

no Last.fm. 68 pessoas responderam ao questionário. Os dados estão sendo tabulados para uma pesquisa em desenvolvimento. 
Atualmente existe uma série de redes emergentes específicas para a música como o MyStrands, o Pandora (que foi fechado para usuários fora dos EUA), Ilike, Spotify, Imeen - que apresentou um crescimento muito grande no último ano - e o Musicovery - que trabalha com as sensações dos gêneros musicais através das cores e do design.

Nossa opção por centralizarmos no Myspace, Last.fm e Blip.Fm se deu a partir de alguns critérios relevantes para o contexto nacional:

a) em função da tradução do site para o português - no caso dos dois primeiros, inclusive destacando que o MySpace possui uma sede em São Paulo desde o ano passado

b) pelo grande acesso e crescimento de usuários brasileiros, mesmo em um sistema como o Blip.fm que ainda não foi traduzido. ${ }^{7}$

Salientamos que essa preocupação com o contexto nacional relaciona-se com o questionário aplicado com os usuários do Last.fm, cujos resultados estão sendo analisados em outro trabalho, mas que pontuam alguns questionamentos no presente artigo. É evidente que muitas dessas práticas se repetem em outros países e contextos. No entanto, como nossos resultados dizem respeito a determinados atores sociais que se encontram em um contexto cultural específico, torna-se necessária a demarcação. Além disso, e a título de exemplo, em abril desse ano, o Last.fm começou a cobrar para quem quiser utilizar o serviço de webrádio para quem morar fora dos Estados Unidos, Inglaterra e Alemanha, o que pode gerar um outro tipo de reconfiguração dos usos da plataforma a partir de um corte econômico e por país.

Os dois primeiros sites de redes sociais cujo enfoque se relacionavam diretamente com a distribuição de música, o Last.fm e o MySpace, foram ambos lançados no ano de 2003, conforme podemos observar na linha do tempo da Figura 1. O MySpace foi fundado em Julho de 2003 por Tom Anderson (ex-aluno da Universidade de Berkeley e da UCLA), e em 2005 foi comprado parcialmente pela by Intermix Media, por 580 milhões de dólares, pela Rupert Murdoch's News Corporation.

\footnotetext{
${ }^{7}$ O site de métricas Alexa.com aponta o Brasil como nono país com mais usuários da plataforma Blip.fm, correspondendo a $1,7 \%$ de usuários e com uma média de 36.881 visitas diárias. Disponível em http://www.alexa.com/data/details/traffic_details/blip.fm 11/02/2009.
} 
O Last.fm foi fundado em 2002 na Inglaterra, embora seu lançamento oficial só tenha acontecido em 2003, e é uma das maiores plataformas sociais de música com mais de 65 milhões de músicas em seu catálogo e 21 milhões de usuários mensais, além de um adicional estimado em 19 milhões de usuários através de aplicativos convergentes com outras plataformas como APIs e widgets (Schäeffer, 2008: 278). O Last.fm foi uma fusão de duas fontes diferentes que aconteceu em 2005: um mashup entre o plugin audioscrobbler e a plataforma social Last.fm que se transformou no nome oficial da comunidade. Em 30 de maio de 2007, ele foi adquirido pela CBS Interactive pelo valor de 280 milhões de dólares. Atualmente ele está disponível em 12 idiomas.

Já o site Blip.fm é bem mais recente e foi lançado em maio de 2008 pela empresa Fuzz ${ }^{8}$, voltada exclusivamente para o mercado musical online. A plataforma de microblog surgiu como "uma forma de sugerir música e compartilhar os pensamentos sobre elas com uma rede de contatos” (Hendrickson, 2008: Online). O Blip.fm rastreia músicas de outros sites como o Seeqpod e dos próprios usuários. A ferramenta tem crescido bastante entre os aficcionados por música (ver FIG.2) e os usuários do Twitter, uma vez que ela permite a integração de ambas as plataformas. Além disso, os "DJs" (como são chamados os usuários) mais populares ganham "props e bottoms" e são identificados com pontos para os outros usuários - estratégias de aumento de visibilidade e reputação.

FIGURA 1 - Linha do tempo de lançamento de alguns dos principais sites de redes sociais

\footnotetext{
8“ Fuzz, the company that created Blip, is a self-described "CRM for bands" - a place where indie artists can set up web presences and manage their relationships with fans. It also features a mixtape maker like Mixwit and Muxtape, but with songs contributed directly by artists themselves" (HENDRICKSON , 2008: Online)
} 


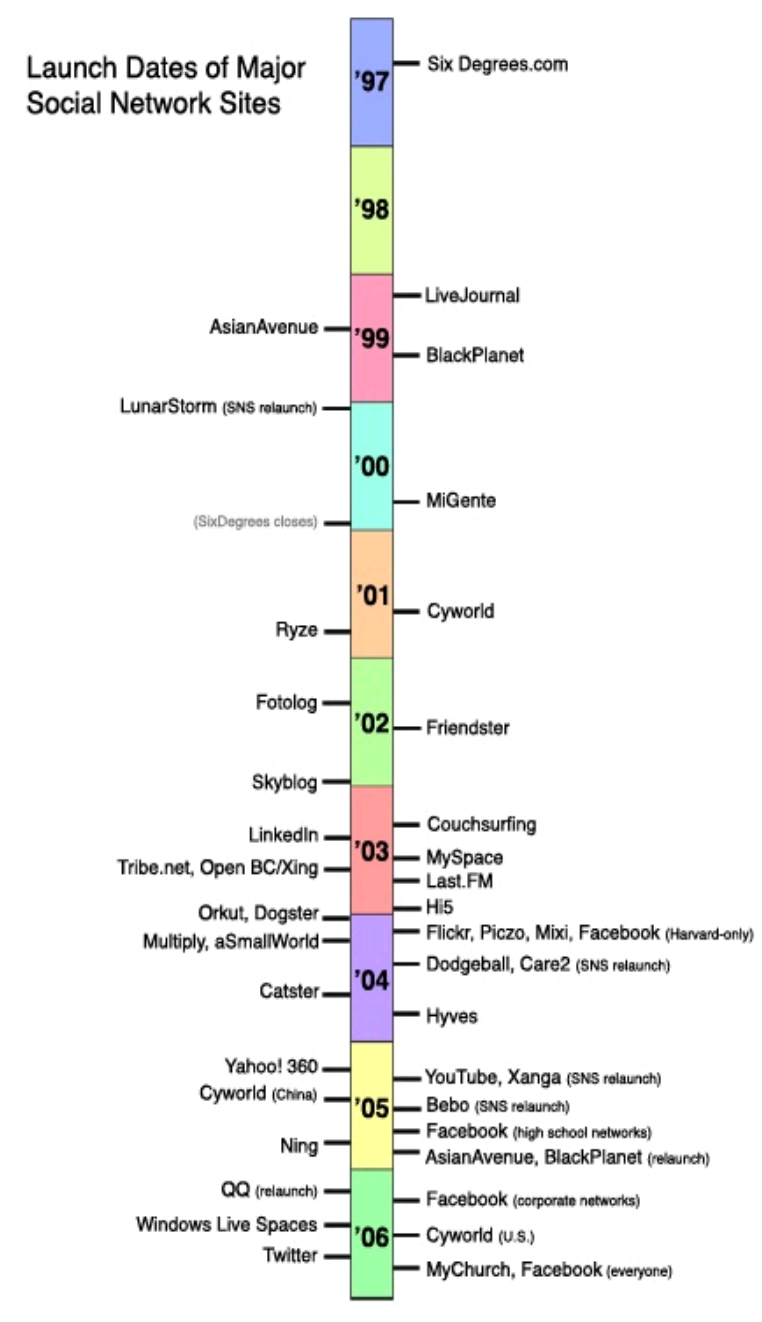

FONTE - BOYD \& ELLISON (2007, Online)

FIGURA 2 - Comparativo de tráfego (percentual diário) entre MySpace, Last.fm e Blip.fm

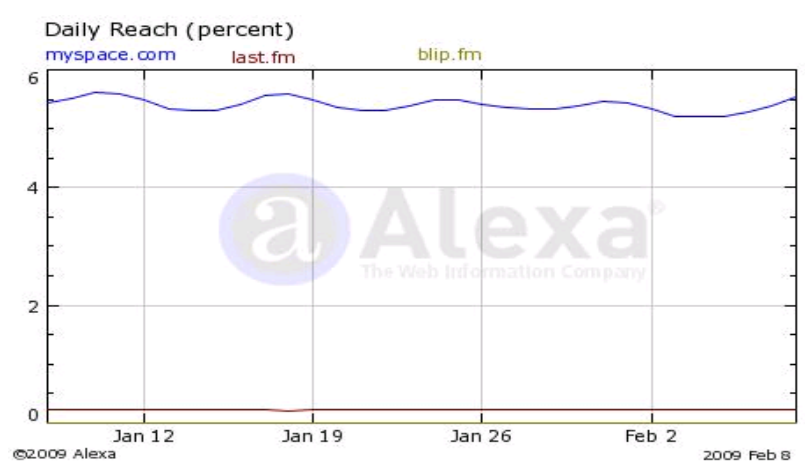

FONTE - ALEXA (http://www.alexa.com/ 10/02/2009) 
As pesquisas sobre a temática dos sites de redes sociais têm crescido juntamente com o surgimento e a apropriação das mesmas. Autores como Donath (2004), Recuero (2005), Boyd (2006), Hodkinson (2007), entre outros, apresentam distintas análises e métodos para os mesmos. "Scholars from disparate fields have examined SNSs in order to understand the practices, implications, culture, and meaning of the sites, as well as users' engagement with them” (Boyd \& Ellison, 2007, Online). No entanto, há poucos estudos que tratem das especificidades das plataformas musicais ou que as relacionem com o consumo musical. Entre eles Amaral (2007a, 2007b e 2009), Amaral e Aquino (2008), Leão e Prado (2007), Accoutier \& Pachet (2007), Baym \& Ledbetter (2008) e Schäefer (2008).

As definições a respeito desse formato de site de relacionamento/sistema/software social/plataforma também são bastante imprecisas. A partir do ponto de vista da computação social e da informação, Accoutier \& Pachet (2007) os observam como sites públicos de banco de dados de música compartilhada ou mesmo mecanismos de dados musicais a partir de "taggeamento" colaborativo. Turbnull, Barrignton e Lanckriet (2008) os descrevem ora enquanto sites de descoberta de música ora enquanto sistemas híbridos de descoberta, recomendação e visualização musical.

Leão \& Prado (2007: 71) optam por uma outra definição, que as aproxima da linguagem radiofônica: "programas que simulam estações de rádios e oferecem a possibilidade de escutar música”. Apesar dos aspectos simuladores da linguagem das estações de rádio estarem presentes explicitamente nos sites, tanto na produção de “tabelas dinâmicas mostrando as músicas mais ouvidas de determinado artista” (Leão \& Prado, 2007: 71), por exemplo, acreditamos que a noção de programa que simula rádio não dá conta da totalidade de fluxos comunicacionais que essas redes permitem aos usuários e nem de outras remixagens midiáticas a partir dos aplicativos.

A estação de rádio enquanto forma particular de comunicação e linguagem se apresenta como apenas um dos elementos constitutivos do perfil, todavia é complementar a todas as outras formas convergentes de participação cultural como as práticas de social tagging (Lamere \& Celma,2007, Amaral \& Aquino, 2008), de nãoinclusão de determinadas músicas enquanto construção identitária através do 
mecanismo de desligamento do rastreador, no caso do Last.fm (Amaral, 2007), e de monitoramento, visualização e mapeamento dos dados musicais a partir do consumo dos usuários, como em alguns projetos que citaremos a seguir.

O projeto de Nepusz (2008) Reconstructing the structure of theworld-wide musical scene with last.fm é um mapa que

representa representa graficamente as mais de quatro milhões de relações de similaridade entre os artistas que constam da base de dados da rede social. Os círculos representam os artistas, bandas e músicos que podem ser encontrados na secção de música do site. As linhas ligam os artistas com sonoridades mais próximas, em função dos hábitos musicais dos utilizadores. Cada estilo musical encontra-se sob a forma de uma cor, tendo em conta as etiquetas associadas pelos utilizadores aos artistas. (CAETANO, 2008: Online).

O mapa foi gerado a partir da API aberta do Last.fm e permite descobrir a localização dos artistas inserindo o nome ou mesmo descobrir os artistas preferidos a partir do nome de tela do usuário do last.fm (FIG 3).

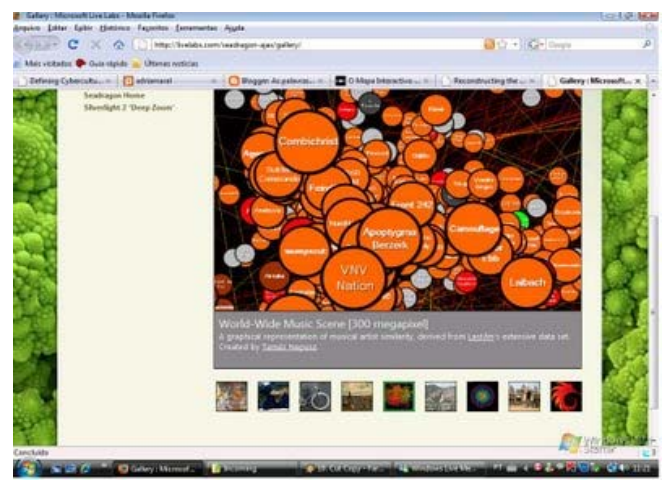

FIGURA 3 - Mapa dos gêneros musicais com base nos dados do usuário adriamaral FONTE - AMARAL (21/11/2008)

Já o trabalho Monitoring and visualizing Last.fm dos artistas e pesquisadores Adjei \& Holland-Cunz (2008) parte de perguntas interrogadas ao sistema para monitorar e visualizar o consumo musical, como, por exemplo, que artista só possui um 
hit, quais comunidades de fãs são mais receptivas ao hip-hop...

By listening to it, the member continuously generates new data, which are saved in the public database being pertinent to it. We observed part of these data for a period of four months and evaluated them in order to find answers to the above questions, for the audience of Last.fm. Our project consists of four parts: 01 »Comparing Fan-Groups«, 02 »Fluctuation of Fans«, 03 »Album-Release« and 04 »Cumulation of Genres«. Within these scopes, we can present most interesting results gained from our observations. All visualizations were realized by using the programming language and integrated development environment Processing 9 .

Esse bancos de dados ajudam a compreender a partir de gráficos e visualizações nossa concepção a respeito desse tipo específico de site de rede social, que está mais próxima do pensamento de autores como Baym \& Ledbetter (2008) e Schäefer (2008), que descrevem o Last.fm, por exemplo, como uma plataforma online em sentido comunicacional mais amplo, no qual são apresentados aspectos implícitos e explícitos de participação.

Last.fm provides several communication platforms for those interested in using the site socially, including writing publiclyvisible messages on one another's profiles in the "shoutbox", sending one another private personal messages, and participating in site-wide discussion forums. (Baym \& Ledbetter, 2008: 06)

Optamos momentaneamente pelo termo plataforma por seu uso relacionado à web e sua multiplicidade de serviços; por seu sentido computacional relacionado ao software e aos sistemas operacionais - plataforma UNIX, por exemplo - e principalmente pela carga simbólica como metáfora relacionada aos meios de transporte e de comunicação - plataformas de veículos como trem ou ônibus ou plataformas como local onde há oportunidade de expressão de ideias, performances e discursos (LONGMAN, 2006: 1251). O exemplo de Shäefer (2008) sobre o Last.fm colabora para compreensão do consumo, da convergência e das trocas e serviços que acontecem no

\footnotetext{
${ }^{9}$ Informações disponíveis emhttp://visualizinglastfm.de/about.html Acesso em 21/11/2008
} 
interior dessas plataformas.

It is an ecosystem where the creativity of developing communities meets the intellectual property of the music industry, but where emerging and independent artist can also promote their music, where event organizers can advertise, and retailers can sell their products, and it furthermore serves as a "third place" where users can meet. Moreover, Last.fm is not limited to the Last.fm website, but spreads out through the application programming interface to any other platform. (SCHÄEFER, 2008: 281)

Contudo, é interessante problematizar essas tênues matrizes conceituais a partir da proposição de Manovich (2008) de inserção desse tipo de estudo no que ele chama de "estudos de software" ou "teorias do software" ${ }^{\text {, }}$ observados sob uma perspectiva cultural e humanista.

I think of software as a layer that permeates all areas of contemporary societies. Therefore, if we want to understand contemporary techniques of control, communication, representation, simulation, analysis, decision-making, memory, vision, writing, and interaction, our analysis can't be complete until we consider this software layer. (MANOVICH, 2008: 10, grifo do autor)

Embora reduzidas por não serem o foco desse trabalho, essas discussões são fundamentais para a compreensão dos fluxos comunicacionais, sociais, estéticos, etc. que acontecem com base nos sites de redes sociais, blogs, wikis e outras ferramentas digitais.

\subsection{Classificação, categorização e colecionismo de gêneros musicais pelos fãs}

Um dos maiores enfoques na literatura sobre o assunto trata-se da análise das classificações e categorizações dos gêneros musicais a partir dessas plataformas de

\footnotetext{
${ }^{10}$ Para o autor, essas ferramentas "are in the center of the global economy, culture, social life, and, increasingly, politics. And this "cultural software" - cultural in a sense that it is directly used by hundreds of millions of people and that it carries "atoms" of culture (media and information, as well as human interactions around these media and information). (MANOVICH, 2008: 3)
} 
música online. Salientamos que dentre os três casos estudados, apenas o Last.fm possibilita a escrita colaborativa de tags definidoras dos gêneros musicais - mas outros sistemas de recomendação como Pandora e Spotify, por exemplo, também permitem esse recurso. O MySpace possui uma variedade mínima de tags que são escolhidas pelo usuário apenas quando ele se cadastra para construir o perfil, como rock, clássico e jazz, por exemplo. Já o Blip.fm não possui nenhuma forma de tagging e seu sistema de recomendação funciona apenas a partir do nome do artista cuja música foi recentemente tocada.

Tais pesquisas indicam uma preocupação com variedade de tags coletadas a partir dos usuários para categorização dos estilos musicais, contribuindo para análise dos usos e formas de colecionismo de música online através do social tagging. Essa discussão é indicada por autores como Lamere (2008) e Turnbull, Barrington e Lanckriet (2008) - que discutem a questão do efeito de "bias de popularidade", seja em termos de gêneros e canções mais populares (que os autores chamam de short-head) e menos populares (cauda longa).

Assim, ocorrem hibridizações intergêneros, que se perpetuam ora pelo uso contínuo das tags propostas pelos usuários - no caso brasileiro, por exemplo, as tags já existentes no sistema são utilizadas por $72 \%$ dos usuários entrevistados - ora pelas próprias relações sociais de "amizade" que se configuram no sistema a partir da constituição do gosto musical, conforme nos indicam Baym \& Ledbetter (2008), possibilitada por medidores comparativos próprios do sistema como o "gostômetro de gosto” ou o aplicativo que mede o nível de "mainstreamness".

Essas práticas são dimensionadas pela organização das buscas e informação sobre um núcleo de estilos musicais - e as possibilidades de recomendação possam ser fatores importante nessa constituição -, pois a partir desses dois elementos (tagging e recomendação) podemos medir/visualizar presença e permanência na plataforma afetando assim a constituição da reputação e mesmo da autoridade (normalmente vinculado aos grupos/subculturas de cunho musical) no sistema.

Tais considerações iniciais constituem uma tentativa de definição e análise desse tipo específico de plataforma cuja lógica opera, em certo sentido, em um misto de micromídia e mídia de nicho (Thornton, 1996) no qual as disputas simbólicas de capital 
subcultural e de DIY entre os fãs (Jenkins, 2006) enquanto curadores desse acervo de memória informativa (Jennings, 2008) - nesse caso entre gêneros musicais - aparecem com força.

\title{
3. Plataformas de música online
}

Descritas algumas das principais características e definições observadas pelos teóricos, passemos então a uma análise do corpus enquanto artefatos culturais cujas significações indicam que "a escuta da música em fluxo revela-se em toda sua transparência, enquanto relações com networks e base de dados, em um continuum de experiências vividas” (Leão \& Prado, 2007: 79).

\subsection{Last.fm}

The Last.fm socio-technical ecosystem oscillates between copyrighted content and the free use of an information system. Last.fm mediates between major players from the music industry and a large number of users, who require additional value to just downloading music. (Schäefer 2008: 281)

O Last.fm (Figura 4) é uma plataforma baseada no compartilhamento e recomendação musical que funciona com estações de rádio, fóruns e sistema de etiquetamento e indexação dos arquivos de música - esse taggeamento é feito pelos próprios usuários (folksonomia), construindo assim uma vasta base de dados sobre artistas dos mais diferentes gêneros musicais - que são analisados a partir do download do plugin audioscrobbler, cuja função é fazer a leitura desses arquivos no computador e/ou ipod pessoal e publicá-las no perfil daquele usuário, conectando-o à anima collectiva da plataforma, com os perfis de amigos e vizinhos.

\begin{abstract}
Sistemas de recomendação têm por objetivo reduzir a sobrecarga de informação da web atual por meio da seleção de conteúdo baseada em preferências pelo usuário. (...) Modelos de recomendação baseiam-se na interseção dos contextos de usuários para estimar uma recomendação, o que tem sido feito através de dados semânticos ou pela análise de redes sociais. (Figueira Filho, Geus e Albuquerque, 2008: 1)
\end{abstract}

Em estudos anteriores, observamos que por ter um contexto segmentado apenas em gêneros e subgêneros musicais, a folksonomia do Last.fm é de tipo estreita 
(Quintarelli, 2005), beneficiando assim o etiquetamento de objetos que não são facilmente encontrados com ferramentas tradicionais e fornecem alvos de audiências, ou seja, pessoas que compartilham vocabulários próprios e que assim podem recuperar os itens de forma mais simples e eficiente. Tal constatação fica ainda mais explícita quando se avalia os dados obtidos pelo questionário, de que $72 \%$ dos entrevistados usam as tags recomendadas pelo sistema em vez de criá-las e que 76\% utilizam sempre a mesma tag para determinado gênero/artista.

Consider the fact that on the music sharing site Last.fm.there are two distinct categories of significant others, namely 'friends' (i.e. contacts) and 'neighbours' (people with whom you share a taste in music on the basis of your digital trail on the site). In other words, the builders of Last.fm have recognised the crucial importance of two very different sociological principles: proximity in social network terms and proximity in sociodiscursive space (musical taste). (Postill, 2008: Online)

Além de artistas e estilos musicais similares, também há recomendações de shows, festas e eventos musicais, a partir de uma base geográfica determinada pela escolha de localização do usuário. Assim, ao me cadastrar como moradora da cidade de Porto Alegre, Londres ou São Paulo, são recomendadas apresentações que acontecerão naquela cidade. Richard Jones, um dos fundadores e CEO da empresa, enfatiza que "recommendation and discovery is key in this space now - and we've been working on this for 6 years, and every day we continue to refine the process” (Jones in MacManus, 2008: Online).

O ecossistema sociotécnico (Schäefer, 2008) ou espaço sociodiscursivo musical (Postill, 2008) também apresenta a relação de proximidade de gostos a partir dos aplicativos de comparação dos artistas mais ouvidos, calculados a partir de um filtro de algoritmo a partir da comparação entre as playlists. Essa proximidade musical aparentemente ampliaria amizades e laços sociais entre os ouvintes (Leão \& Prado, 2007), no entanto, o estudo de Baym \& Ledbetter (2008) aponta que essa plataforma não leva a laços fortes - salvo se for utilizado de forma integrada a outras plataformas.

Outro importante fator para análise apontado na literatura sobre sites de redes sociais diz respeito à personalização. No last.fm - que passou por um redesign no ano 
passado $^{11}$ - ainda não é possível alterar as configurações do perfil e além da escolha estratégica da foto e algumas informações como link para o site ou blog pessoal há poucos rastros além das listagens musicais e da tagcloud de estilos musicais. A única mudança possível, em termos de design, é a opção de escolher entre a cor vermelha e a preta para o cabeçalho (Figura 4). Assim, observamos que a "identidade" do avatar nessa plataforma é constituída a partir do gosto musical, sendo um sistema pouco conversacional $^{12}$ e muito mais de visibilidade e colecionismo, no qual talvez a construção da reputação possa ser observada nos aspectos de mídia de nicho e micromídia (Thornton, 1996) - acontecendo muito mais pela adesão e legitimação do "saber" dos "pares musicais", encontrados no sistema e na questão de multiplexidade midiática $^{13}$ (Haythornthwaite, 2005), ou seja, na passagem e integração de uma mídia à outra: o usuário adiciona um amigo no last.fm, mas no entanto interage conversacionalmente com ele via outra ferramenta.

FIGURA 4 - Perfil do usuário Daniel HDR

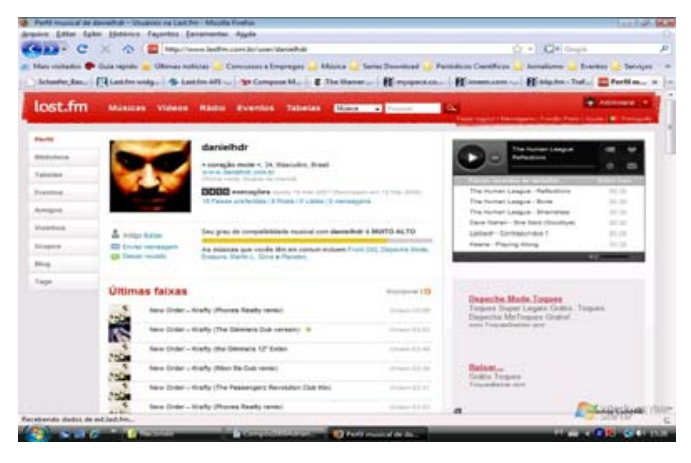

FONTE - LAST.FM - http://www.lastfm.com.br/user/danielhdr Acesso em 10/02/2009

\footnotetext{
${ }^{11}$ Ver entrevista com $\quad$ condador fund http://www.readwriteweb.com/archives/interview_with_lastfm_founder_part3_design_features.php Acesso em 03/10/2008.

${ }^{12}$ Observa-se pouco uso dos blogs e da "shoutbox" (que seria uma caixa de comentários) nas práticas dos usuários. Baym e Ledbetter(2008) também afirmam pouca interação na plataforma per se.

${ }^{13}$ A noção de multiplexidade de mídias desenvolvida por Haythornthwaite (2005) tem suas origens na teoria sociológica e relaciona a questão numérica de ferramentas midiáticas ao fortalecimento dos laços sociais.
} 


\subsection{MySpace}

Apesar de não funcionar como sistema de recomendação, o MySpace Music é um dos principais agregadores de artistas/fãs na rede, servindo tanto como plataforma de lançamento de novos artistas, como fonte de informação e de instrumento de divulgação daqueles já consagrados que vêem no site uma possibilidade de relacionamento direto com a base de fãs. Uma prática comum observada anteriormente (2009) é a do redirecionamento do link das páginas pessoais dos artistas para o perfil. Todavia, analistas do mercado da música como Dubber (2007) não recomendam essa estratégia como eficiente para a divulgação online, uma vez que a promoção multiplataforma congregaria uma "identidade distribuída" e se torna mais eficiente.

A popularidade da plataforma no país - que desde 2007 foi traduzida para o português é grande no contexto do entretenimento e do acesso (Ver os dados na Tabela 1). No âmbito das mídias tradicionais ela é utilizada como fonte para o jornalismo musical, indicando datas de turnês e/ou lançamentos de álbuns.

O design do MySpace é completamente livre e pode ser completamente customizado pelo usuário. Em uma leitura dos perfis de variadas “cenas musicais” é comum percebermos a repetição dos "atores sociais”, como "top friends” (mais amigos) na página inicial. Observam-se algumas práticas de inserção de determinado artista num contexto musical específico. É o caso, por exemplo, da banda Aire' nterre (FIG.5), cujos perfis escolhidos de artistas e fãs dispostos na primeira página (os “top friends”) - portanto a mais acessada - são em sua maioria de outras bandas e artistas independentes cujos estilos musicais são muito próximos dos gêneros nos quais o projeto está inserido. Também são encontrados nessa disposição fãs ativos no cenário de música eletrônica alternativa. As tags que descrevem o perfil (industrial/alternativa/gótica) complementam qualquer dúvida sobre a "identidade musical” de um usuário que o acesse pela primeira vez. Até mesmo o template de cores escuras apresenta a consciência intencional de atingir uma microaudiência definida.

FIGURA 5 - Perfil do usuário Airen'terre 


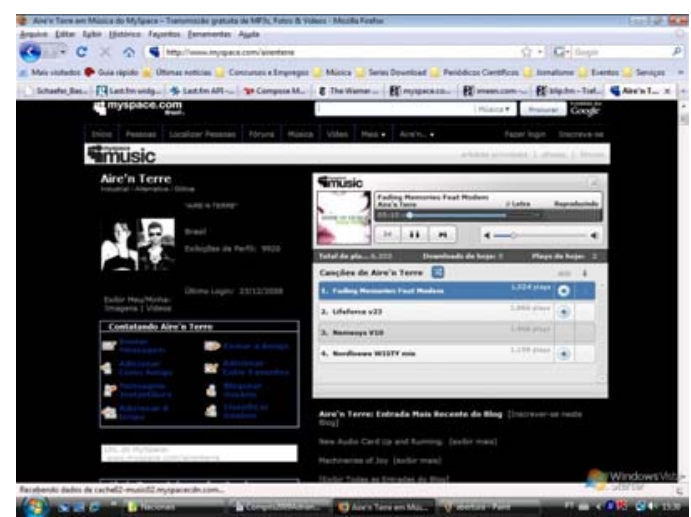

FONTE - MYSPACE - http://www.myspace.com/airenterre Acesso 10/02/2009

Assim, algumas estratégias de construção de identidades musicais emergem de forma mais amplificada, atribuindo maior visibilidade aos processos de colecionismo e da constituição de uma base de dados musicais para um determinando fandom - cujo consumo e produção de conteúdo gera uma ampla gama de conhecimentos nas múltiplas mídias (Jenkins, 2006), indicando uma conscientização explícita a respeito de uma audiência segmentada, que retroalimentará as informações nas mais variadas plataformas além do MySpace.

\subsection{Blip.fm}

O Blip.fm é uma plataforma de microblog que permite o compartilhamento de músicas e comentários sobre elas. A idéia do site é uma espécie de "seja você mesmo um DJ de 150 caracteres”. Vários aplicativos têm sido desenvolvidos e o site tem agregado um considerável número de ouvintes nos últimos meses, inclusive brasileiros (Tabela 1). Esse crescimento ocorreu muito em função da integração via Twitter ${ }^{14}$.

Since May, its developers have added several distinguishing features, such as badges for members who accumulate a horde of followers. The idea behind these badges is to recognize the most popular DJs and identify them for new members. In the same vein, members can also give each other "props" points whenever they like the songs that others have shared. (HENDRICKSON, 2008: Online)

As interações sociais e a reputação são constituídas através de algumas práticas

\footnotetext{
${ }^{14}$ http://twitter.com Acesso em 10/01/2009
} 
como a distribuição de "props” - espécie de pontos de parabenização pela escolha da música que um usuário dá ao outro; dos reblips - quando um usuário posta a mesma música de um dos DJs da sua lista; e através do ato de responder diretamente a um usuário utilizando o símbolo @ na frente do nome do usuário. Apesar dessas interações, o layout se mantém fixo e não há possibilidade de personalização do perfil.

Blip users post 'blips' to tracks - and as with Twitter, others can follow your Blips and listen to what you've posted. It's micromusic-blogging. Now that Twitter has become so popular, there is a whole micro-economy built around Twitter - with multiple companies providing every different style of twitter client_that you could possibly want, for just about any platform Twitter has enabled this economy by providing a rich set of web services around their system that any client can tap into. Blip is hoping to do the same thing. They are providing a rich set of web services around their core that allows any third party to interact with the Blip service. (LAMERE, 2009, Online)

O Blip.fm retoma a ideia de DJ "jukebox", funcionando como uma rede de estações de rádios personalizadas a partir do compartilhamento de subjetividades musicais. As recomendações aparecem logo após a postagem da música, indicando que outros usuários da plataforma também compartilharam aquele artista.

\section{Considerações Finais}

No presente artigo, discutimos a conceitualização das plataformas de música online a partir de diferentes estudos sobre sites de redes sociais, observando a construção identitária dos perfis e as formas de consumo musical. Para tanto, comparamos, ainda que preliminarmente, três diferentes plataformas, a partir dos eixos da classificação e recomendação; da divulgação e relação social entre fãs e artistas e da postagem de microconteúdos musicais a partir da interação entre perfis. Na tabela abaixo, procuramos de forma condensada apresentar alguns dos fluxos comunicacionais e as características que transpareceram com mais evidência em nossa análise comparativa e que podem contribuir para futuras discussões sobre a temática.

TABELA 1

Fluxos comunicacionais que caracterizam as plataformas de música online 


\begin{tabular}{|c|c|c|c|}
\hline Características & Last.fm & MySpace Music & Blip.fm \\
\hline Recomendação & $\begin{array}{l}\text { Recomendação funciona } \\
\text { pelas tags de gêneros } \\
\text { musicais, de artistas e pela } \\
\text { localização geográfica do } \\
\text { usuário (no caso dos } \\
\text { shows) }\end{array}$ & Não possui um modelo & $\begin{array}{l}\text { O sistema de recomendação } \\
\text { se dá apenas pelo nome do } \\
\text { artista/música. }\end{array}$ \\
\hline Tags & $\begin{array}{l}\text { Usuários criam tags } \\
\text { livremente }\end{array}$ & $\begin{array}{l}\text { Número limitado de tags } \\
\text { que o usuário marca ao } \\
\text { construir o perfil. }\end{array}$ & $\begin{array}{l}\text { Não possui sistema de } \\
\text { taggeamento organizado. } \\
\text { Contudo, a prática de } \\
\text { utilização hashtags é } \\
\text { similar à do Twitter, } \\
\text { colocando o símbolo \# na } \\
\text { frente da palavra. }\end{array}$ \\
\hline Principais funções & $\begin{array}{l}\text { Social tagging } \\
\text { Recomendação } \\
\text { Rastreamento (scrobbling) } \\
\text { Escuta } \\
\text { Geração de playlists } \\
\text { Disponibilização e } \\
\text { compartilhamento de } \\
\text { músicas }\end{array}$ & $\begin{array}{l}\text { Disponibilização e } \\
\text { compartilhamento de } \\
\text { músicas } \\
\text { Escuta } \\
\text { Expressão pessoal } \\
\text { Interação fãs-artistas }\end{array}$ & $\begin{array}{l}\text { Escuta, disponibilização e } \\
\text { compartilhamento de } \\
\text { músicas } \\
\text { Recados para os amigos } \\
\text { Informações subjetivas } \\
\text { sobre a música }\end{array}$ \\
\hline Laços sociais & $\begin{array}{l}\text { Para Baym \& Ledbetter } \\
\text { (2008) são fracos e apenas } \\
\text { se amplificam na } \\
\text { convergência com outras }\end{array}$ & $\begin{array}{l}\text { Relações podem variar } \\
\text { entre fortes e fracas, mas } \\
\text { observa-se fortes laços } \\
\text { entre fãs-artistas e/ou } \\
\text { participantes de uma } \\
\text { mesma subcultura } \\
\text { musical. }\end{array}$ & $\begin{array}{l}\text { Aspectos conversacionais } \\
\text { (através dos reblips, props e } \\
\text { respostas) }\end{array}$ \\
\hline Design e formato & $\begin{array}{l}\text { Poucas informações, sejam } \\
\text { visuais ou textuais no } \\
\text { perfil } \\
\text { Cores: Fundo branco e } \\
\text { cinza } \\
\text { Estrutura fixa de tabelas e }\end{array}$ & $\begin{array}{l}\text { Variável de acordo com } \\
\text { o perfil, no entanto } \\
\text { mantém o player das } \\
\text { músicas em cima e a } \\
\text { exibição dos perfis } \\
\text { amigos embaixo. } \\
\text { Normalmente excesso }\end{array}$ & $\begin{array}{l}\text { Microblogging } \\
\text { Perfil apresenta foto e } \\
\text { poucas informações } \\
\text { Postagens das músicas em } \\
\text { ordem cronológica reversa }\end{array}$ \\
\hline
\end{tabular}




\begin{tabular}{|c|c|c|c|}
\hline & menus & visual. & $\begin{array}{l}\text { Postagem dos amigos } \\
\text { Cores: fundo azul e branco } \\
\text { com algumas cores - } \\
\text { laranja e amarelo em } \\
\text { detalhes mais significativos }\end{array}$ \\
\hline $\begin{array}{l}\text { Integração e } \\
\text { convergência }\end{array}$ & $\begin{array}{l}\text { APIs e Mashups } \\
\text { Blogs, Youtube }\end{array}$ & $\begin{array}{l}\text { Pouca } \\
\text { Youtube }\end{array}$ & $\begin{array}{l}\text { Twitter, Friendfeed,e Jaiku; } \\
\text { blogs e Last.fm. }\end{array}$ \\
\hline Mobilidade & $\begin{array}{l}\text { Integração com o Google } \\
\text { Android } \\
\text { Scrobbling para iPhone }\end{array}$ & Não há referências & Postagem via celular \\
\hline Subculturas & $\begin{array}{l}\text { Engajamento a partir das } \\
\text { playlists, das tags e } \\
\text { tagclouds dos perfis, } \\
\text { recomendações e } \\
\text { comparações de } \\
\text { compatibilidades musicais, } \\
\text { foruns. }\end{array}$ & $\begin{array}{l}\text { Engajamento a partir da } \\
\text { construção visual-sonora } \\
\text { do perfil e dos “amigos” } \\
\text { relacionados }\end{array}$ & $\begin{array}{l}\text { O engajamento pode ser } \\
\text { feito parcialmente através } \\
\text { das músicas e } \\
\text { recomendações - na } \\
\text { localização de outros } \\
\text { usuários e das “props”(que } \\
\text { são bônus e congratulações } \\
\text { pela música postada) }\end{array}$ \\
\hline $\begin{array}{l}\text { Participação dos } \\
\text { usuários brasileiros }\end{array}$ & $\begin{array}{l}22^{\circ} \text { país que mais utiliza a } \\
\text { ferramenta (número de } \\
\text { usuários brasileiros } \\
\text { corresponde a } 0,8 \% \text { do } \\
\text { total). Fonte: Alexa.com } \\
\text { Apesar da audiência } \\
\text { brasileira não ser tão } \\
\text { representativa, leva-se em } \\
\text { consideração que é um site } \\
\text { de nicho, cujos perfis } \\
\text { normalmente estão entre } \\
\text { "early adopters" e "heavy } \\
\text { users”. }\end{array}$ & $\begin{array}{l}16^{\circ} \text { país que mais utiliza } \\
\text { a ferramenta (o número } \\
\text { de usuários brasileiros } \\
\text { corresponde a } 0,5 \% \text { do } \\
\text { total). É o } 62^{\circ} \text { site mais } \\
\text { acessado do país. Fonte: } \\
\text { Alexa.com }\end{array}$ & $\begin{array}{l}9^{\circ} \text { país que mais utiliza a } \\
\text { ferramenta (o número de } \\
\text { usuários brasileiros } \\
\text { corresponde a } 1,7 \% \text { do } \\
\text { total). Fonte: Alexa.com }\end{array}$ \\
\hline
\end{tabular}

FONTE - AMARAL, 2009

Enfatizamos que essas características não devem ser tomadas como uma tentativa de sistematização rígida das plataformas, mas sim como uma observação preliminar dos modos de consumo musical no âmbito dos sites de relacionamento, no qual detectamos tanto práticas há muito constituídas no offline - como no caso do ato da recomendação, que já era feita tanto pelo boca-a-boca quanto através das mídias massivas, mas que nesse caso apresenta o elemento do 
cálculo aperfeiçoado; como nas novas formas de busca de informações musicais e do relacionamento entre fãs, artistas e a música.

Dessa forma, as relações entre o consumo musical e a plataforma apresentam-se através de tais fluxos que indicam as apropriações do conhecimento e divulgação musical por parte dos usuários a partir de apropriações das plataformas, seja em relação à constituição, classificação e curadoria de uma memória musical; seja através da consciência de atingir uma determinada audiência de nicho ou subcultural e finalmente na construção de visibilidade e reputação através dos aspectos conversacionais e/ou de integração (por APIs) das plataformas.

\section{Referências bibliográficas}

ACOUTURIER, J.J., PACHET, F. How Much Audition Involved in Everyday Categorization of Music? Disponível em: http://www.jj-aucouturier.info/papers/COG-2007.pdf , 2007.

ADJEI, C., HOLLAND-CUNZ, N. Monitoring and visualizing Last.fm. Disponível em: http://visualizinglastfm.de/index.html. Ago. 2008. Acesso em: 17/01/2009.

AMARAL, A. Fãs-usuários-produtores: uma análise das conexões musicais nas plataformas sociais MySpace e Last.fm. In: PERPETUO, I.F., SILVEIRA, S.A. (orgs). O futuro da música depois da morte do CD. SP: Momento Editorial, pp.91-106. 2009. Disponível em: http://www.futurodamusica.com.br

A. Subculturas e cibercultura(s). Para uma genealogia das identidades de um campo.

Revista FAMECOS, Porto Alegre, n.37,pp.38-44, Dez. 2008. Disponível em http://www.pucrs.br/famecos/pos/revfamecos/37/adriana.pdf

A. Categorização dos gêneros musicais na Internet - Para uma etnografia virtual das práticas comunicacionais na plataforma social Last.FM. In: FREIRE FILHO, J., HERSCHMANN, M. (Org.). Novos rumos da cultura da mídia. Indústrias, produtos e audiências. 01 ed. Rio de Janeiro: Mauad, 2007, p. 227-242.

A estética cibergótica na Internet: música e sociabilidade na comunicação do MySpace. Revista Comunicação, Mídia e Consumo, n.09, São Paulo, p.p. 75-87, 2007b.

AMARAL, A, AQUINO, M.C. Práticas de folksonomia e social tagging no Last.fm. In: Anais do IHC 08 - VIII Simpósio Brasileiro de Fatores Humanos em Sistemas Computacionais, Porto Alegre, 2008. Disponível em: http://www.inf.pucrs.br/ihc2008/ptbr/assets/files/Praticas Folksonomia Social Tagging_Lastfm.pdf

BAYM, N., LEDBETTER, A. Tunes that bind?: Predicting Friendship Strength in a MusicBased Social Network. In: Anais da Aoir 9- Internet Research 9.0, Copenhagen, Denmark, October 2008. Disponível em http://www.onlinefandom.com/wpcontent/uploads/2008/10/tunesthatbind.pdf Acesso em: 10/11/2008. 
BOYD, danah. "Friends, Friendsters, and MySpace Top 8: Writing Community Into Being on Social Network Sites.” First Monday 11:12, December.http://www.firstmonday.org/issues/issue11_12/boyd/index.html. 2006.

BOYD, D. M., \& ELLISON, N. B. Social network sites: Definition, history, and scholarship. Journal of Computer-Mediated Communication, 13(1), article 11. 2007. Disponível em: http://jcmc.indiana.edu/vol13/issue1/boyd.ellison.html . Acesso em 01 março 2008.

BRUNO, F. Monitoramento, classificação e controle nos dispositivos de vigilância digital. Revista FAMECOS, v. 36, p. 1-7, 2008.

BRUNS, A. Blogs, Wikipedia, Second Life and Beyond: From production do produsage. NY: Digital Formations, 2007.

BURKE, P. Modernidade, cultura e estilos de vida. In: BUENO, M.L., CAMARGO, L. Cultura e consumo. Estilos de vida na contemporaneidade. SP: Editora Senac, 2008.

CAETANO, M. O mapa interactivo do Last.fm. Remixtures, 20/11/2008. Disponível em: http://remixtures.com/2008/11/o-mapa-interactivo-da-lastfm/ Acesso em: 21/11/2008.

DONATH, J., boyd, d. Public displays of connection. BT Technology Journal, 22 (4), 71-82. 2004.

DUBBER, Andrew. New music strategies. The 20 things you must know about music online. E-book, Junho 2007. Disponível em: http://newmusicmanagement.com/wpcontent/uploads/2008/06/nms.pdf . Acesso em 28/06/2008.

ECK, D. Et al. Automatic generation of social tags for music recommendation. 2007. Disponível em http://www.iro.umontreal.ca/ eckdoug//papers/2007_nips.pdf Acesso em 05/02/2009.

FIGUEIRA FILHO, F.M., GEUS, P.L, ALBUQUERQUE, J.P. Sistemas de recomendação e interação na Web Social. In: Anais do VII Simpósio Brasileiro de Fatores Humanos em Sistemas Computacionais, PUCRS, Porto Alegre, 2008. Disponível em: http://www.inf.pucrs.br/ihc2008/pt-

br/assets/files/Sistemas_de_Recomendacao_e_Interacao_na_Web_Social.pdfAcesso em: 15/01/2009.

HAYTHORNTHWAITE, C. Social networks and Internet connectivity effects. Information, Communication, \& Society, 8 (2), 125-147. 2005.

HENDRICKSON, M. Twitter for music. TechCrunch, 12/05/2008. Disponível em: http://www.techcrunch.com/2008/05/12/twitter-for-music/ Acesso em: 08/02/2009.

HODKINSON, P. Interactive Online Journals and Individualisation. New Media and Society, Vol $92007 . \quad$ (4), 20 disponível http://www.paulhodkinson.co.uk/publications/hodkinsonnmsarticle.pdf Acesso em 05/12/2008.

HUBERMAN, B., ROMERO, D., WU, F. Social networks that matter: Twitter under the microscope. First Monday, V.14, n.1, 5 Jan. 2009. Disponível em: http://firstmonday.org/htbin/cgiwrap/bin/ojs/index.php/fm/article/view/2317/2063 Acesso em 10/01/2009. 
JENKINS, Henry. Fans, bloggers and gamers. Exploring participatory culture. NY: New York University Press, 2006.

JENNINGS, D. 2008. Fans will be the most comprehensive curators. In: Net, blogs and rock n'roll, 23/05/2008. Disponível em: http://www.netblogsrocknroll.com/2008/05/fans-will-beth.html Acesso em 03/10/2008.

LAMERE, P. The Blip.fm API. Music Machinery. Disponível em: http://musicmachinery.com/2009/02/10/the-blipfm-api/ Acesso em 11/02/2009.

LEÃO, L., PRADO, M. Música em fluxo: programas que simulam rádios e a experiência estética em redes telemáticas. Revista Líbero, SP, Ano X, n.20, pp. 69-79. Dez. 2007.

LIU, H. Social network profiles as taste performances. Journal of Computer-Mediated Communication, vol. 13 (1), artigo 13, 2007. Disponível em http://jcmc.indiana.edu/vol13/issue1/liu.html Acesso em 05/07/2008.

LONGMAN, Dictionary of Contemporary English. Essex: Pearson/Longman, 4a.ed., 2006.

MACMANUS, R. Interview with Last.fm founder Richard Jones: Part 1, The Competition. ReadWriteWeb. 01/10/2008. Disponível em: http://www.readwriteweb.com/archives/interview with lastfm founder richard jones part1.ph p Acesso em 03/10/2008.

MANOVICH, L. Software takes command. 2008. Disponível em: http://lab.softwarestudies.com/2008/11/softbook.html

NAONE, Erica. A brief history of microblogging. Technology Review. Set/Out. 2008. Disponível em: https://www.technologyreview.com/files/18810/forward.pdf Acesso em 10/11/2008.

NEPUSZ, T. Reconstructing the structure of theworld-wide musical scene with last.fm. Disponível em: http://sixdegrees.hu/last.fm/index.html Acesso em 21/11/2008.

PINHEIRO, M. Subjetivação e consumo em sites de relacionamento. Revista Comunicação, Mídia e Consumo. SP, v.5, Nov. 2008. pp.103-121. Disponível em: http://revcom2.portcom.intercom.org.br/index.php/comunicacaomidiaeconsumo/article/viewFile 15462/4984 Acesso em: 05/02/2009.

POSTILL, J. From activist networks (1990s) to social networks sites (2000s). 14/09/2008. Disponível em: http://johnpostill.wordpress.com/2008/09/14/from-activist-networks-1990s-tosocial-network-sites-2000s/

QUINTARELLI, E. Folksonomies: power to the people. In: ISKO Italy-UniMIB Meeting, Milan, Jun. 2005. Disponível: http://www.iskoi.org/doc/folksonomies.htm

RECUERO, Raquel. Redes Sociais no Ciberespaço: Uma proposta de Estudo. In: XXVIII INTERCOM - Congresso Brasileiro de Ciências da Comunicação, 2005, Rio de Janeiro. Anais da XXVIII INTERCOM, 2005.

SCHONFELD, E. Last.fm needs more than a redesign to catch up to Imeen. TechCrunch, 15/08/2008. Disponível em: http://www.techcrunch.com/2008/08/15/lastfm-needs-more-than-aredesign-to-catch-up-to-imeem/ Acesso em: 18/08/2008. 
SCHÄEFER, M. Bastard culture! User participation and the extension of cultural industries. Utrecht: All Print Utrecht, Tese de Doutorado, Utrecht University, 2008. Disponível em: http://mtschaefer.net/media/uploads/docs/Schaefer_Bastard-Culture.pdf Acesso em: 29/01/2009.

SIBILIA, P. O show da vida íntima na internet: blogs, fotologs, videologs, orkut e webcams. In: CAIAFA, Janice; ELHAJJI, Mohammed. (Org.). Comunicação e Sociabilidade: cenários contemporâneos. Rio de Janeiro: Mauad, 2007.

THORNTON, Sarah. Club cultures: music, media and subcultural capital. Connecticut: Wesleyan University Press, 1996.

TURNBULL, D., BARRINGTON, L., LANCKRIET, G.(2008) Five approaches to colleting tags for music. In: ISMIR 2008 - Session 2c - Knowledge Representation, Tags, Metadata. Disponível em http://ismir2008.ismir.net/papers/ISMIR2008_128.pdf Acesso em 21/11/2008.

\section{Sites:}

ALEXA.COM - http://www.alexa.com

BLIP.FM - http://www.blip.fm

LAST.FM- http://www.last.fm

MYSPACE.COM - http://www.myspace.com 